\title{
The RNAi Pathway Initiated by Dicer-2 in Drosophila
}

\author{
K. Kim, ${ }^{*}$ Y.S. LeE, ${ }^{\dagger}$ D. Harris, ${ }^{*}$ K. Nakahara, ${ }^{\dagger}$ AND R.W. CartheW* \\ "Department of Biochemistry, Molecular Biology and Cell Biology, Northwestern University, Evanston, \\ Illinois 60208; Division of Biotechnology, College of Life Sciences and Biotechnology, Korea University, \\ Seoul, South Korea 136-713; ${ }^{*}$ Graduate School of Agriculture, Hokkaido University, Sapporo 060-8589, Japan
}

\begin{abstract}
Injection or expression of double-stranded RNA (dsRNA) in Drosophila serves as a trigger that causes cells to specifically cleave homologous mRNA transcripts. Our approach is to identify essential components of the RNA interference (RNAi) mechanism by isolating and characterizing mutations that cause the RNAi response to be abnormal. These studies have thus far led to the identification of seven genetic loci that encode proteins acting at various steps in the RNAi process. We have molecularly identified several of these proteins. Two are members of the Dicer family. Dicer-1 and Dicer-2 are required for short interfering RNA (siRNA)-directed mRNA cleavage by facilitating distinct steps in the assembly of the RNA-induced silencing complex (RISC). AGO2 is a RISC component that both carries out transcript cleavage and facilitates RISC maturation. Other factors appear to function as regulators of RISC assembly rather than as core factors for RNAi.
\end{abstract}

\section{SECTION THEMES}

Small regulatory RNAs have important roles in a variety of physiological and developmental processes (Zamore 2001). How the generation of such RNAs imparts changes in cell physiology and gene expression is still not well understood. The intracellular machinery used to interpret and implement the effects conveyed by the siRNAs are being actively investigated in a wide variety of experimental systems, using both biochemical and genetic approaches. Biochemical studies with Drosophila and mammalian extracts have led to the identification of proteins that bind to or modify siRNAs (Caudy et al. 2002; Martinez et al. 2002; Caudy and Hannon 2004). Although some of these interactions suggest potential mechanisms of gene regulation, the identities and functions of numerous factors are still unclear.

Genetic screens in Caenorhabditis elegans, Arabidopsis thaliana, and Neurospora crassa have yielded numerous mutant strains with defects in RNAi, and in some cases, the individual mutations were shown to impair specific phases of the RNAi response (Cogoni and Macino 1999; Tabara et al. 1999; Fagard et al. 2000). Biochemical experiments with tissue culture cell extracts have identified several components of the RNAi machinery (Harborth et al. 2001). Drosophila melanogaster embryo and cell extracts have also been employed to dissect the in vitro RNAi pathway (Haley et al. 2003; Lee et al. 2004). Despite these advances, no system has been developed that has fully harnessed the combined power of largescale genetics and in vitro mechanistic analysis. Only Drosophila currently offers the combination of sophisticated genetic tools and a well-developed in vitro RNAi system. Accordingly, in vitro analyses using extracts from RNAi-defective Drosophila strains present a unique opportunity to more precisely define the pathway that leads to gene regulation in response to siRNAs.

Gene expression is silenced by siRNAs in Drosophila through cytoplasmic mRNA transcript degradation, typical of the classically defined RNAi response (Kennerdell and Carthew 1998, 2000). In some cases, as in the maturing oocyte, RNAi activity can be correlated with the translational state of the target transcript (Kennerdell et al. 2002). It suggests that the RNAi machinery is limited to detecting and/or degrading only actively translated mRNAs or that both RNAi and translation independently rely upon some common mechanism of transcript licensing. Although a targeted transcript apparently disappears in toto, the first step in its destruction is a single cleavage of the transcript phosphodiester backbone at a site complementary to one strand of an effector siRNA. This event is followed by degradation of the $5^{\prime}$ and $3^{\prime}$ cleavage products by the exosome and other degradation machineries (Orban and Izaurralde 2005). In this paper, we focus on recent progress in understanding the means by which transcripts are targeted for cleavage and, in particular, the use of genetic analysis to reveal aspects of the intracellular pathway that transforms an siRNA into a gene regulator.

\section{A Way to Identify RNAi Factors through Genetics}

The simplest way to screen for RNAi mutants is to isolate recessive mutants that are viable and fertile but are defective for RNAi. This approach has been used for many genetic screens to isolate RNAi mutants in various model systems. However, much of the RNAi mechanism could have critical links to other processes that are essential for viability or fertility. If so, then the majority of mutants that perturb RNAi would be recessive-lethal or sterile, and would be missed in a screen for viable mutants.

To get around this problem, we created a situation in which flies are heterozygous for a mutation, but their compound eyes are composed of cells that are homozygous mutant. We then looked for mutations that perturb RNAi activity in the compound eye (Fig. 1). We first carried out construction of a Drosophila strain in which eye- 


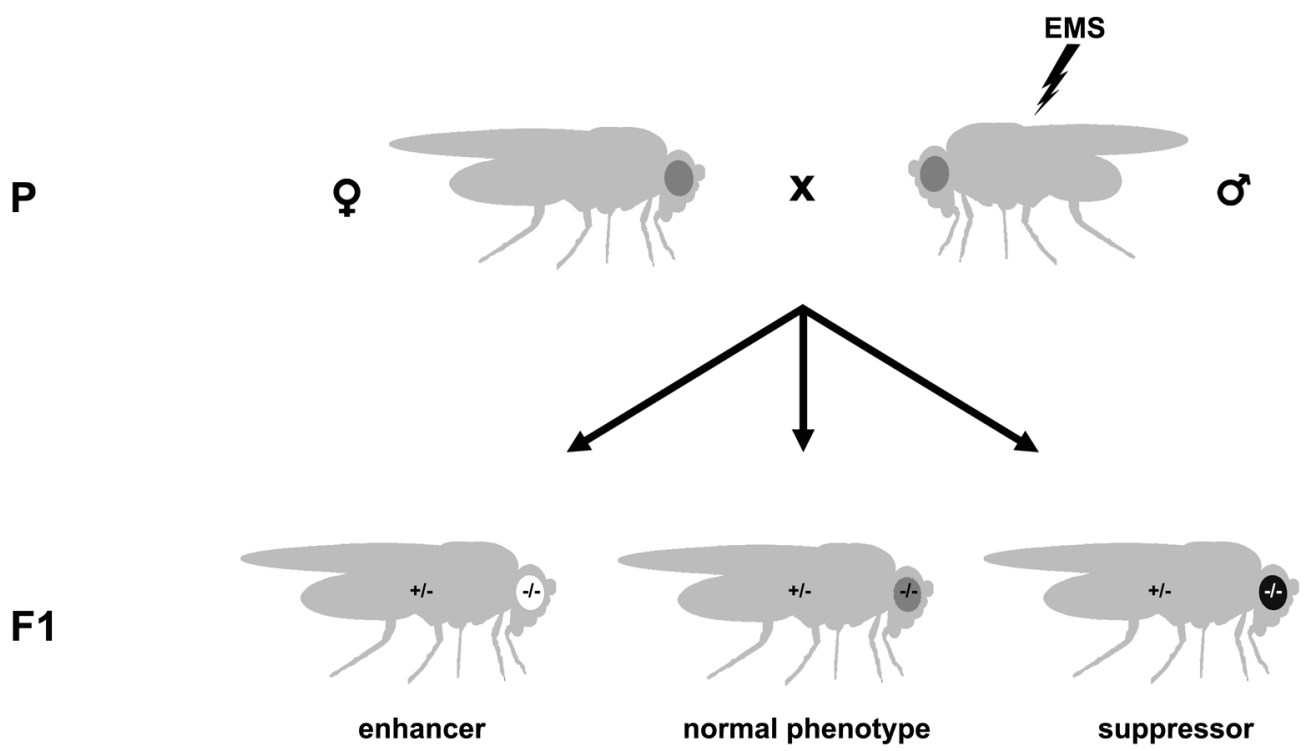

Figure 1. Summary of the genetic screen for mutants with perturbed RNAi activity. RNAi-mediated silencing of the white gene is triggered by the presence of a transgene GMR-wIR, which expresses white dsRNA. The result is an adult fly with partially pigmented eyes (shown here as gray eyes). Males of the parental (P) generation are mutagenized with ethanemethane sulfonate (EMS), and then mated to nonmutagenized females. Their offspring, the $\mathrm{F}_{1}$ generation, are heterozygous mutant and carry one copy of GMR-wIR. However, eye cells carry one chromosome arm that has recombined between sister chromosomes such that the arm is homozygous mutant. If the homozygous-mutant eye cells have normal RNAi, the eye color appears silenced (gray). If the mutant cells have impaired RNAi, the eye color appears dark, and if the mutant cells have enhanced RNAi, the eye color appears white.

specific RNAi is easily monitored (Lee and Carthew 2003; Lee et al. 2004). We chose the white gene as an RNAi target based on several criteria. The white gene encodes an $\mathrm{ABC}$ transporter required cell-autonomously to pigment the adult compound eye. A white $e^{+}$eye is dark red in color, whereas a null white mutant is completely white. This phenotype is easily scored, affording easy and rapid phenotype screening. Moreover, a semiquantitative relationship exists between expression level and phenotype such that activity can be ordered by a phenotypic series: red $\rightarrow$ brown $\rightarrow$ orange $\rightarrow$ yellow $\rightarrow$ white. To generate RNAi against white, a single exon of white was placed in a tail-to-tail orientation downstream from the eye-specific GMR promoter, and this snapback construct was transformed into white ${ }^{+}$flies to create transgenic lines that had a white loss-of-function phenotype (Lee and Carthew 2003). GMR-wIR lines bearing one copy of the transgene had pale orange eyes.

To create flies with homozygous mutant eyes, we used the FLP/FRT system to induce site-specific mitotic recombination (Golic and Lindquist 1989). FLP recombinase was expressed under the control of the eyeless promoter $(e y F L P)$, which is exclusively active throughout the proliferative phase of eye development (Newsome et al. 2000). EyFLP induces recombination between FRT elements on sister chromosomes during cell division, so almost all eye tissue becomes homozygous by the end of the proliferative phase. The nonmutagenized FRT chromosome was marked with the cell-death transgene GMR-hid, which kills retinal cells during metamorphosis (postproliferative). Any retinal cell possessing even one copy of GMR-hid will die, thus leaving only retinal cells homozygous for the mutage- nized chromosome. Since the remaining cells compensate in number for the missing ones by further division, the size and morphology of eyes engineered in this fashion are basically normal.

Animals with one copy of GMR-wIR were examined for mutant eyes that had variant white gene activity. Seven genes were identified in the screen. The products of four of the seven loci were identified by other means as being core RNAi factors.

\section{Dicer-2: An RNase III Enzyme with RISC Assembly Functions}

One of the four loci identified in our screen corresponds to the Dicer-2 gene. The Dicer-2-encoded protein (Dicer-2) is synthesized as a $200-\mathrm{kD}$ protein. The aminoterminal region contains a DExH helicase domain, whereas the carboxy-terminal region contains two tandem RNase III catalytic domains and a single dsRNAbinding domain. The Dicer-2 protein has been shown to have RNase-III-type activity in vitro, and this activity appears to produce siRNAs from dsRNA substrates (Bernstein et al. 2001). Moreover, this activity appears to be essential for Dicer-2 function in vivo. Three lines of evidence suggest that Dicer-2 has enzymatic preference to process siRNAs rather than microRNAs (miRNAs). First, purified Dicer-2 protein exhibits strong siRNA-producing activity and weak miRNA-producing activity (Provost et al. 2002; Myers et al. 2003). Second, Dicer-2 mutants show specific deficits in siRNA production in vivo. Third, Dicer-2 mutants are completely viable and fertile, whereas loss of miRNAs results in complete loss of viability (Lee et al. 2004). 
What domains in Dicer-2 are responsible for siRNA production? Point mutation of key catalytic residues within each RNase III domain abolishes siRNA production (Lee et al. 2004). Thus, these domains are necessary. In addition, the DExH helicase domain is important for siRNA production; point mutation of residues within the ATP-binding pocket greatly reduces activity. Why is a helicase activity essential? It might be needed to displace the enzyme from the dsRNA substrate upon cleavage, thus enabling multiple rounds of enzymatic activity. Interestingly, the other Dicer in Drosophila, Dicer-1, lacks a DExH helicase domain and has poor siRNA productivity in vivo.

Dicers act as catalysts for processing small regulatory RNAs including siRNA and miRNA. Do Dicers have any other function in the RNAi reaction? We found that Dicer-2 is required in vivo for transcript destruction in response to artificially supplied siRNA (Lee et al. 2004). This implies that Dicer-2 functions in a second step in addition to its known function as a small RNA processor. We took advantage of the robust biochemistry of RNAi afforded by Drosophila to characterize the RNAi defects of the Dicer-2 mutant. We found that extracts derived from Dicer-2 mutants exhibit severe defects in mRNA target cleavage activity triggered by an added siRNA. This suggests that Dicer-2 protein functions in the effector phase of RNAi, again consistent with in vivo results. To explore the biochemical basis for the target mRNA cleavage defect in the mutant extract, we examined the assembly of RISC complexes with labeled siRNA.

When siRNA is incubated with extract derived from Drosophila embryos, several discrete complexes are seen by native gel electrophoresis (Pham et al. 2004). Dicer-2 protein directly contacts siRNA in these complexes, as determined by UV cross-linking experiments. Moreover, the complexes fail to form when siRNA is incubated with extract derived from Dicer-2 mutant embryos (Pham et al. 2004). Thus, Dicer-2 associates with siRNA in vitro to form stable complexes. What are the relationships of the different complexes to each other? Pulse-chase experiments show that one of the complexes serves as a precursor to all of the other complexes. Further analysis has revealed that the precursor complex is a heterotrimer composed of siRNA, Dicer-2, and the R2D2 protein (Pham and Sontheimer 2005). For this reason, we call this complex the R2D2-Dicer-2 initiator (RDI). RDI is thought to initiate the assembly of the other complexes. One of the other complexes has many features expected of the RISC effector, although it is far larger $(\sim 80 \mathrm{~S})$ than any RISC yet described. The complex contains siRNA in a single-stranded state, it copurifies with known RISC factors (TSN, FMR, VIG, and $\mathrm{AGO} 2$ ), and it binds and cleaves targeted mRNAs (Pham et al. 2004). For these reasons, we refer to this complex as holo-RISC. Interestingly, holo-RISC also contains Dicer-2 protein, although unlike RDI, it does not have Dicer-2 in intimate association with siRNA. The RDI and holo-RISC appear to be the beginning and end of an ordered biochemical pathway for RISC assembly (Fig. 2). Dicer-2 does not simply transfer siRNAs to

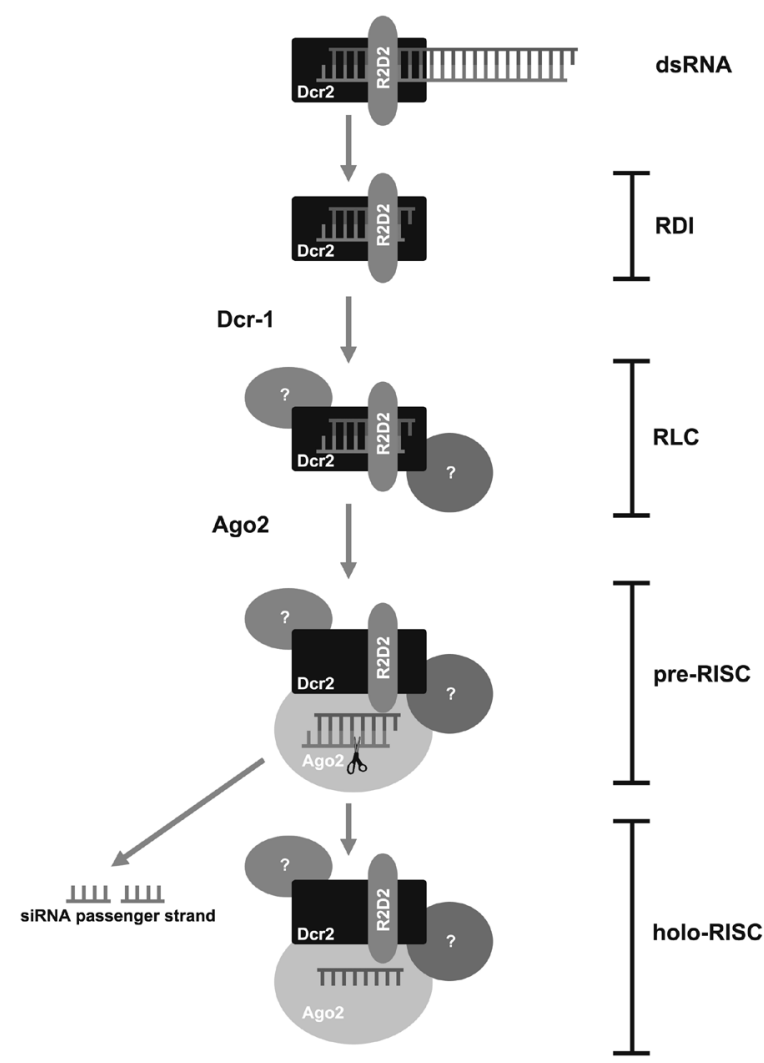

Figure 2. Model for the Dicer-2-mediated RNAi pathway (see text for details).

a distinct RISC complex, but rather assembles into RISC along with the siRNAs, indicating that its role extends beyond the initiation phase of RNAi.

\section{R2D2: A dsRNA-binding Protein Partner of Dicer-2}

The Dicer-2 protein is not required for viability or fertility; its loss appears to affect only RNAi activity. We were therefore encouraged to seek mutations in other genes that would lead to the loss of RNAi and, like Dicer-2, would not lead to lethal or sterile phenotypes. One such gene that emerged from our mutagenesis screen encodes the R2D2 protein. R2D2 contains two tandem dsRNA-binding domains (dsRBDs). Loss of R2D2 function results in strong derepression of white gene silencing by GMR-wIR. The R2D2 protein heterodimerizes with Dicer-2, but Dicer2 does not require R2D2 to cleave dsRNA into siRNA (Liu et al. 2003; Liu et al. 2006). What is R2D2 doing in association with Dicer-2? When R2D2 is mutated, siRNA is not efficiently loaded into holo-RISC, and the assembly of the RDI complex is not observed in the mutant. This observation correlates with the detection of R2D2 as a subunit of RDI. Therefore, R2D2 and Dicer-2 seem to connect the initiator and effector phases of RNAi. The C. elegans RDE-4 protein has a structure similar to that of R2D2, and RDE-4 associates with the nematode Dicer in vitro (Tabara et al. 2002). Similar orthologs have been detected in complex with Dicers of vertebrate species. Thus, the role of R2D2 is probably conserved in other organisms. 


\section{AGO2 Has an Essential Role in RISC Assembly and Function}

Our screen identified a viable mutation that partially derepressed white gene activity. On the basis of a variety of genetic data, we have identified the mutation as an allele of Ago2. Several previous studies have indicated a role for Ago2 in RNAi. AGO2 protein is a physical subunit of RISC, and loss of Drosophila Ago2 gene activity results in a failure to form holo-RISC (Okamura et al. 2004). Furthermore, structural studies of an archaebacterial AGO protein found that it resembles RNase $\mathrm{H}$, an RNase that cleaves the RNA strand of RNA/DNA hybrid duplexes (Lingel et al. 2003; Song et al. 2004). These studies suggested that AGO proteins might directly cleave the target RNA strand of a target:siRNA hybrid duplex in RISC. Indeed, purified human $\mathrm{AGO} 2$ protein possesses target RNA cleavage activity, and mutation of key catalytic residues results in loss of this activity (Liu et al. 2004).

There is a sequence motif, GxDV, within the PIWI domain of eukaryotic AGO proteins that is highly conserved (Parker et al. 2004). The aspartate residue is critical to coordinate the nucleophile that catalyzes target RNA cleavage. The mutation $\operatorname{Ago}^{V 966 M}$ found in our screen changes the GxDV motif into GxDM (Kim et al. 2006). This valine residue is highly conserved among all AGO proteins. Modeling of a GxDM variant of the AGO structure with PyMol reveals a steric change in the integrity of the structure at the catalytic site. We suspect that the V-to-M mutation in AGO2 alters nucleophile coordination required for normal catalysis. Consistent with this notion, western blot analysis reveals that there is a normal level of AGO2 protein present in the mutant even though target RNA cleavage activity is reduced 16fold in the mutant.

To test if $A g o 2^{V 966 M}$ is nevertheless able to form holoRISC, we analyzed RISC assembly by native gel electrophoresis of siRNA-protein complexes. Typically, native gels exhibit the RDI and holo-RISC complexes, as well as two intermediate complexes (Kim et al. 2006). One of these is the RISC-loading complex (RLC) and the other is the pre-RISC complex. The assembly pathway in vitro follows RDI to RLC to pre-RISC to holo-RISC (Fig. 2). Formation of each complex appears to have discrete requirements for different protein factors. For example, Dicer-2 is required to form RDI, and hence RLC, preRISC, and holo-RISC (Pham et al. 2004). In contrast, AGO2 protein is required to form pre-RISC and holoRISC but not RDI and RLC (Kim et al. 2006). When we tested the $\mathrm{Ago} 2^{\text {V966M }}$ mutant, both pre-RISC and holoRISC are present, but there is 2-fold less holo-RISC and an apparent buildup of pre-RISC. The fact that $\mathrm{AgO} 2^{\mathrm{V} 966 \mathrm{M}}$ has a 2-fold decrease in the level of holo-RISC and a 16fold decrease in holo-RISC activity suggests that the mutant protein has impaired catalytic activity, as predicted. However, why should the mutant show a partial block in the pre-RISC to holo-RISC assembly step?

The answer to this question requires us to understand the differences between pre-RISC and holo-RISC. When we visualize the conformation of siRNA associated with various complexes formed in the assembly reaction, RDI,
RLC, and pre-RISC complexes contain duplex siRNA (Kim et al. 2006). In contrast, holo-RISC contains singlestranded siRNA, the other strand presumably lost from the complex. Thus, key steps in converting pre-RISC to holo-RISC are siRNA strand unwinding and preferential strand retention (Fig. 2). However, Ago $2^{V 966 M}$ complexes predominantly contain duplex siRNA. Thus, AGO2 catalytic activity appears to stimulate conversion of siRNA duplex into single-stranded siRNA within higher-order RISC complexes (Kim et al. 2006), possibly by strand unwinding or facilitating strand loss. We found that the mutant shows defective siRNA-unwinding comparable to its defective holo-RISC-forming activity. Recent in vitro studies have shown that unwinding of the siRNA duplex is accompanied by cleavage of the ejected siRNA strand during holo-RISC formation (Matranga et al. 2005; Rand et al. 2005). In the $A g o 2^{V 966 M}$ mutant, the rate of siRNA strand cleavage is strongly reduced. These data support a model where siRNAs are initially loaded as duplexes into AGO2-containing pre-RISC. The catalytic activity of $\mathrm{AGO} 2$ is then required to cleave one strand of the duplex, stimulating it to dissociate from the uncleaved strand and ultimately dissociate from RISC. The end result is a RISC retaining one siRNA strand, fully competent to down-regulate transcript targets.

If AGO2-dependent catalysis is obligatory for complex conversion, then we might have expected a more profound assembly block in the Ago $2^{V 966 M}$ mutant. One explanation is that conversion of pre-RISC to holo-RISC occurs by an independent mechanism when AGO2 is unable to cleave siRNA. Alternatively, conversion is promoted by several factors working in parallel, each factor augmenting conversion. For example, it is conceivable that AGO2-dependent cleavage of one siRNA strand acts to facilitate an active unwindase or to prevent stable reassociation of the strands after an unwindase has acted. The identity of such an unwindase remains unknown. The RNA helicase Armitage is required for RISC assembly (Tomari et al. 2004), but it seems to act in the RLC to preRISC step of the pathway before unwinding has occurred (data not shown).

\section{RISC Assembly and Dicer-1}

Formation of the RLC complex from RDI is poorly understood. It is a rapid ATP-independent step, forming a relatively unstable complex. RLC contains duplex siRNA in association with Dicer-2 and R2D2. The identities of other RLC subunits are yet unknown. Interestingly, another Drosophila Dicer protein has been implicated in RLC formation. Dicer-1 is a $220-\mathrm{kD}$ protein that lacks the amino-terminal DExH helicase domain typically found in Dicer proteins. The Dicer-1 protein has RNase-III-type activity in vitro, but this activity appears to be specific for miRNA synthesis and not siRNA synthesis (Lee et al. 2004). Consistent with this observation, Dicer-1 mutants show specific deficits in miRNA production in vivo, resulting in complete loss of viability.

Despite this preference for miRNA processing, Dicer-1 is also active in the siRNA pathway. Dicer- 1 mutants show derepressed white gene silencing in the presence of the 
$G M R-w I R$ trigger, and these mutants are also defective in silencing targets that are triggered by exogenous siRNA. Thus, Dicer-1 is required downstream from siRNA production. Our biochemical analysis indicates that Dicer-1 acts as an activator of RLC formation. Extracts from Dicer1 mutants fail to form stable RLC complexes in vitro. Moreover, UV cross-linking experiments implicate Dicer1 protein as a factor in close association with siRNA in RLC complexes (Pham et al. 2004). Further biochemical studies will be required to determine whether Dicer-1 is actually an obligatory subunit of RLC. The available data do, however, permit the conclusion that the level of Dicer1 can be a limiting step in RLC formation. A similar complex to RLC has been identified in C. elegans. It consists of Dcr-1, Rde-4, the AGO protein Rde-1, and one or two RNA helicases, Drh-1 and Drh-2 (Tabara et al. 2002).

\section{Regulation of RISC Assembly: Links between RLC and RISC}

The RLC consists of Dicer-2/R2D2 closely associated with siRNA duplex and yet unknown factors. The siRNA must undergo a radical transformation in that it changes to a single-stranded form that is closely associated with AGO2 rather than Dicer-2. Conversion of RLC to preRISC involves this latter step. Several lines of evidence suggest that this step might be rate-limiting and subject to regulation both in vitro and in vivo. First, in vitro, the rate of pre-RISC formation is very slow relative to RDI and RLC formation. Second, formation is stimulated by ATP, suggesting that the process is more complicated than mere intermolecular association/dissociation. Third, genetic analysis has identified putative regulators of RISC assembly that appear to affect the RLC to pre-RISC step.

Whereas Dicer-1, Dicer-2, R2D2, and Ago2 mutations show impaired silencing of a white target gene in vivo, mutations in two other genes show enhanced silencing of white by GMR-wIR. Our data implicate these genes as encoding repressors of the RNAi response (Y.S. Lee et al., unpubl.). Indeed, biochemically both repressors appear to inhibit production of holo-RISC by attenuating the rate of pre-RISC formation. These data have several implications. They argue that RNAi is under some endogenous negative control for reasons that are as yet unclear. They argue that the RLC to pre-RISC step is an effective step in which regulation can be exerted, and they argue that RNAi can be engineered to be stronger using facile methods to manipulate fly strains.

\section{CONCLUDING REMARKS}

The experimental advantages of being able to examine mutant phenotypes in a tissue that is dispensable for viability and fertility ensure that new insights will continue to emerge from studies of RNAi in Drosophila. In particular, the utility of genetic screens for enhancers and suppressors of RNAi to identify components of the pathway has been established and has encouraged us to start similar endeavors for other pathways. We believe that the success of our previous screens depended on our ability to create a situation where the RNAi-responsive tissue (the eye) contained completely mutant cells while the rest of the animal contained heterozygous cells. This ability to identify mutations in heterozygous individuals permits not only the screening of large numbers of individuals, but also the identification of genes with other roles during development whose total loss of function would be lethal. Finally, the advantage of performing biochemical assays with extracts from normal and mutant strains in parallel has permitted an unprecedented ability to pinpoint the molecular activities of those products identified in our screens.

\section{ACKNOWLEDGMENTS}

We thank the National Institutes of Health, FRAXA Foundation, and the Damon Runyon Foundation for their support.

\section{REFERENCES}

Bernstein E., Caudy A.A., Hammond S.M., and Hannon G.J. 2001. Role for a bidentate ribonuclease in the initiation step of RNA interference. Nature 409: 363.

Caudy A.A. and Hannon G.J. 2004. Induction and biochemical purification of RNA-induced silencing complex from Drosophila S2 cells. Methods Mol. Biol. 265: 59.

Caudy A.A., Myers M., Hannon G.J., and Hammond S.M. 2002. Fragile X-related protein and VIG associate with the RNA interference machinery. Genes Dev. 16: 2491.

Cogoni C. and Macino G. 1999. Gene silencing in Neurospora crassa requires a protein homologous to RNA-dependent RNA polymerase. Nature 399: 166.

Fagard M., Boutet S., Morel J.B., Bellini C., and Vaucheret H. 2000. AGO1, QDE-2, and RDE-1 are related proteins required for post-transcriptional gene silencing in plants, quelling in fungi, and RNA interference in animals. Proc. Natl. Acad. Sci. 97: 11650.

Golic K.G. and Lindquist S. 1989. The FLP recombinase of yeast catalyzes site-specific recombination in the Drosophila genome. Cell 59: 499 .

Haley B., Tang G., and Zamore P.D. 2003. In vitro analysis of RNA interference in Drosophila melanogaster. Methods 30: 330 .

Harborth J., Elbashir S.M., Bechert K., Tuschl T., and Weber K. 2001. Identification of essential genes in cultured mammalian cells using small interfering RNAs. J. Cell Sci. 114: 4557.

Kennerdell J.R. and Carthew R.W. 1998. Use of dsRNA-mediated genetic interference to demonstrate that frizzled and frizzled 2 act in the wingless pathway. Cell 95: 1017.

2000. Heritable gene silencing in Drosophila using double-stranded RNA. Nat. Biotechnol. 18: 896.

Kennerdell J.R., Yamaguchi S., and Carthew R.W. 2002. RNAi is activated during Drosophila oocyte maturation in a manner dependent on aubergine and spindle-E. Genes Dev. 16: 1884.

Kim K., Lee Y.S., and Carthew R.W. 2006. Conversion of preRISC to holo-RISC by Ago2 during assembly of RNAi complexes. RNA (in press).

Lee Y.S. and Carthew R.W. 2003. Making a better RNAi vector for Drosophila: Use of intron spacers. Methods 30: 322.

Lee Y.S., Nakahara K., Pham J.W., Kim K., He Z., Sontheimer E.J., and Carthew R.W. 2004. Distinct roles for Drosophila Dicer-1 and Dicer-2 in the siRNA/miRNA silencing pathways. Cell 117: 83 .

Lingel A., Simon B., Izaurralde E., and Sattler M. 2003. Structure and nucleic-acid binding of the Drosophila Argonaute 2 PAZ domain. Nature 426: 465.

Liu J., Carmell M.A., Rivas F.V., Marsden C.G., Thomson J.M., Song J.J., Hammond S.M., Joshua-Tor L., and Hannon G.J. 2004. Argonaute 2 is the catalytic engine of mammalian RNAi. Science 305: 1437. 
Liu Q., Rand T.A., Kalidas S., Du F., Kim H.E., Smith D.P., and Wang X. 2003. R2D2, a bridge between the initiation and effector steps of the Drosophila RNAi pathway. Science 301: 1921.

Liu X., Jiang F., Kalidas S., Smith D., and Liu Q. 2006. Dicer-2 and R2D2 coordinately bind siRNA to promote assembly of the siRISC complexes. RNA 12: 1514.

Martinez J., Patkaniowska A., Urlaub H., Luhrmann R., and Tuschl T. 2002. Single-stranded antisense siRNAs guide target RNA cleavage in RNAi. Cell 110: 563.

Matranga C., Tomari Y., Shin C., Bartel D.P., and Zamore P.D. 2005. Passenger-strand cleavage facilitates assembly of siRNA into Ago2-containing RNAi enzyme complexes. Cell 123: 607.

Myers J.W., Jones J.T., Meyer T., and Ferrell J.E. 2003. Recombinant Dicer efficiently converts large dsRNAs into siRNAs suitable for gene silencing. Nat. Biotechnol. 21: 324.

Newsome T.P., Asling B., and Dickson B.J. 2000. Analysis of Drosophila photoreceptor axon guidance in eye-specific mosaics. Development 127: 851.

Okamura K., Ishizuka A., Siomi H., and Siomi M.C. 2004. Distinct roles for Argonaute proteins in small RNA-directed RNA cleavage pathways. Genes Dev. 18: 1655.

Orban T.I. and Izaurralde E. 2005. Decay of mRNAs targeted by RISC requires XRN1, the Ski complex, and the exosome. RNA 11: 459.

Parker J.S., Roe S.M., and Barford D. 2004. Crystal structure of a PIWI protein suggests mechanisms for siRNA recognition and slicer activity. EMBO J. 23: 4727.

Pham J.W. and Sontheimer E.J. 2005. Molecular requirements for RNA-induced silencing complex assembly in the Drosophila RNA interference pathway. J. Biol. Chem. 280: 39278.

Pham J.W., Pellino J.L., Lee Y.S., Carthew R.W., and Sontheimer E.J. 2004. A Dicer-2-dependent 80s complex cleaves targeted mRNAs during RNAi in Drosophila. Cell 117: 83 .

Provost P., Dishart D., Doucet J., Frendewey D., Samuelsson B., and Radmark O. 2002. Ribonuclease activity and RNA binding of recombinant human Dicer. $E M B O J .21: 5864$.

Rand T.A., Petersen S., Du F., and Wang X. 2005. Argonaute2 cleaves the anti-guide strand of siRNA during RISC activation. Cell 123: 621.

Song J.J., Smith S.K., Hannon G.J., and Joshua-Tor L. 2004. Crystal structure of Argonaute and its implications for RISC slicer activity. Science 305: 1434

Tabara H., Yigit E., Siomi H., and Mello C.C. 2002. The dsRNA binding protein RDE-4 interacts with RDE-1, DCR-1, and a DExH-box helicase to direct RNAi in C. elegans. Cell 109: 861.

Tabara H., Sarkissian M., Kelly W.G., Fleenor J., Grishok A., Timmons L., Fire A., and Mello C.C. 1999. The rde-1 gene, RNA interference, and transposon silencing in C. elegans. Cell 99: 123.

Tomari Y., Du T., Haley B., Schwarz D.S., Bennett R., Cook H.A., Koppetsch B.S., Theurkauf W.E., and Zamore P.D. 2004. RISC assembly defects in the Drosophila RNAi mutant armitage. Cell 116: 831.

Zamore P.D. 2001. RNA interference: Listening to the sound of silence. Nat. Struct. Biol. 8: 746. 


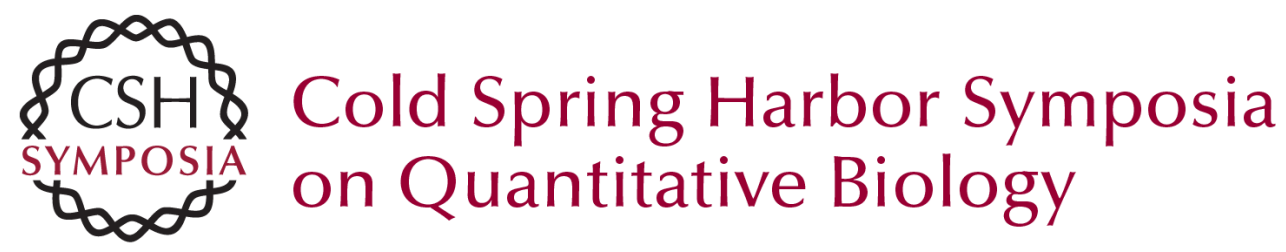

\section{The RNAi Pathway Initiated by Dicer-2 in Drosophila}

K. KIM, Y.S. LEE, D. HARRIS, et al.

Cold Spring Harb Symp Quant Biol 2006 71: 39-44

Access the most recent version at doi:10.1101/sqb.2006.71.008

References This article cites 33 articles, 13 of which can be accessed free at: http://symposium.cshlp.org/content/71/39.full.html\#ref-list-1

\section{License}

Email Alerting Receive free email alerts when new articles cite this article - sign up in Service the box at the top right corner of the article or click here. 